\title{
Matrix Quantization of Turbulence 1
}

\author{
Emmanuel Floratos
}

Department of Physics, Univ. of Athens, GR-15771 Athens, Greece

Institute of Nuclear Physics, N.C.S.R. Demokritos, GR-15310, Athens, Greece

mflorato@phys.uoa.gr

\begin{abstract}
Based on our recent work on Quantum Nambu Mechanics [Axenides \& Floratos (2009)], we provide an explicit quantization of the Lorenz chaotic attractor through the introduction of Non-commutative phase space coordinates as Hermitian $N \times N$ matrices in $R^{3}$. For the volume preserving part, they satisfy the commutation relations induced by one of the two Nambu Hamiltonians, the second one generating a unique time evolution. Dissipation is incorporated quantum mechanically in a self-consistent way having the correct classical limit without the introduction of external degrees of freedom. Due to its volume phase space contraction it violates the quantum commutation relations. We demonstrate that the Heisenberg-Nambu evolution equations for the Matrix Lorenz system develop fast decoherence to $\mathrm{N}$ independent Lorenz attractors. On the other hand there is a weak dissipation regime, where the quantum mechanical properties of the volume preserving non-dissipative sector survive for long times.
\end{abstract}

\footnotetext{
${ }^{1}$ Based on invited talks delivered at: Fifth Aegean Summer School, "From Gravity to Thermal Gauge theories and the AdS/CFT Correspondance", September 2009, Milos, Greece; the Intern. Conference on Dynamics and Complexity, Thessaloniki, Greece, 12 July 2010; Workshop on "AdS4/CFT3 and the Holographic States of Matter", Galileo Galilei Institute, Firenze, Italy, 30 October 2010.
} 


\section{Motivation}

The study of turbulence is a very difficult and interesting subject in various fields of science and engineering since it is very common in nature [Landau \& Lifshitz(1987)]. In modern fundamental physics it has been discussed in extremes scales and temperatures ranging from cosmology for its important role in the determination of the distribution of voids and the distribution of peculiar velocities of galactic clusters around them Gibson \& Schild (2010) to the thermalization process of quark-gluon plasma in heavy ion collisions [Asakawa et al.(2007)] as well as to the non-gaussianities in the velocity spectrum of liquid helium vortex interactions [Van Sciver (2009)].

We are interested at the question of turbulence in quantum dissipative systems. There, dissipation deforms the non-commutative phase space to classical phase space and the basic question is about the spectra of relaxation times to classical behavior. From the point of view of dissipation the problem is equivalent to the one of open Hamiltonian Weiss (2008) or non-Hamiltonian systems Tarasov (2008). Moreover turbulent behavior presupposes a fluid structure for the system with a superimposed mechanism for the creation and annihilation of vortices [Landau \& Lifshitz(1987)].

The phase space noncommutativity in Quantum mechanics at times $t=0$ is given by the Canonical Commutation Relations(CCR)

$$
\left[Q_{i}, P_{j}\right]=\imath \hbar \delta_{i j} \quad t=0
$$

where $Q_{i}, P_{i} \quad i=1,2 \cdots$ are conjugate canonical pairs of position and momentum for a system with $n$ degrees of freedom. In the Heisenberg picture the operators develop in time according to the Heisenberg-Hamilton eqs. of motion

$$
\begin{aligned}
\imath \hbar \dot{Q}_{i} & =\left[Q_{i}, H\right] \quad i=1, \ldots, n \\
\imath \hbar \dot{P}_{i} & =\left[P_{i}, H\right]
\end{aligned}
$$

with the CCRs preserved in time.

If dissipation is added linearly by hand then the Heisenberg eqs. become [Weiss (2008)

$$
\begin{aligned}
\imath \hbar \dot{Q}_{i} & =\left[Q_{i}, H\right] \\
\imath \hbar \dot{P}_{i} & =\left[P_{i}, H\right]-\imath \hbar \Gamma P_{i} \quad i=1, \ldots, n
\end{aligned}
$$

with the commutator decaying exponentially in time

$$
\left[Q_{i}(t), P_{j}(t)\right]=\exp (-\Gamma t) \quad\left[Q_{i}(0), P_{j}(0)\right]
$$

One of the most well studied toy models,as mechanisms for the onset of chaos, is the Lorenz chaotic attractor Eckmann \& Ruelle (1985)]. Through the work of Ruelle and 
Takens an explosion of theoretical and experimental work followed on the general roads to turbulence through the study of strange attractors in dynamical systems Viana (2000).

If one is interested in the problem of turbulence in quantum systems such as, liquid Helium, Bose-Einstein condensates(BEC) or the quark-gluon plasma, or more generally in the quantum regime of the Big Bang and its later epochs, one must create a framework to quantize the Navier-Stokes equation as well as its relativistic generalization Landau (1941).

In the present talk we address this question in the case of a toy model for turbulence , the Lorenz attractor. Nevertheless we can pose the problem for more general dynamical systems. We now proceed to tackle the following issues Tarasov (2008)

a) Is it possible to quantize volume preserving flows in fluid dynamics of the form

$$
\frac{d x^{i}}{d t}=f^{i}(\vec{x}) \quad i=1, \cdots, n
$$

for general non-Hamiltonian dynamical systems which upon introduction of dissipation lead to strange attractors?

b) The same problem but with the inclusion of dissipation that renders the system chaotic with strange attractors.

In the present talk, we will answer both questions in the affirmative by expressing incompressible flow equations in terms of the Nambu n-brackets with $\mathrm{n}$ being the dimensionality of the dynamical phase space $x^{1}, x^{2}, \cdots, x^{n}$. For the case of the Lorenz attractor $n=3$. We will show how to quantize the volume preserving Nambu flow equations, which are associated to the Lorenz attractors and as a second step we consider also the inclusion of dissipation. For more information on the Lorenz and Rössler attractors as well as their equivalents we refer the reader to our recent work Axenides \& Floratos (2010), Axenides (2011) .

The quantization scheme we propose is analogous to the standard Heisenberg quantization of classical mechanics leading to a unique time evolution for the system. As a concrete example we will treat the quantization of the Lorenz strange attractor by $\mathrm{N} \times \mathrm{N}$ Hermitian matrices implementing our flow decomposition methodology into nondissipativedissipative components.

\section{Nambu vs Hamilton's Mechanics}

In Hamilton's mechanics phase space is an even dimensional symplectic manifold $\left(M_{2 n}\right)$ $n=1,2, \cdots$ thus posssessing the structure of a Poissonian manifold Arnold (1978)]. We consider two such examples:

A) The Real Plane $M_{2}=R^{2}$ with Poisson bracket

$$
\{f, g\}=\epsilon_{i j} \partial^{i} f \partial^{j} g \quad f, g \in C^{\infty}\left(R^{2}\right)
$$


which for the coordinates $x_{1}, x_{2}$ gives

$$
\left\{x_{i}, x j\right\}=\epsilon_{i j} \quad i, j=1,2
$$

Given a Hamiltonian $H \in C^{\infty}\left(R^{2}\right)$ a flow vector field is defined

$$
L_{H}=\frac{\partial H}{\partial x_{2}} \frac{\partial}{\partial x_{1}}-\frac{\partial H}{\partial x_{1}} \frac{\partial}{\partial x_{2}}, \quad \dot{f}=L_{H} f
$$

For different Hamiltonians, we have the integrability condition

$$
\left[L_{H_{1}}, L_{H_{2}}\right]=L_{\left\{H_{1}, H_{2}\right\}}
$$

B) $M=S^{2}$ the 2-dimensional sphere embedded isometrically in $R^{3}$, $x_{1}^{2}+x_{2}^{2}+x_{3}^{2}=1 \quad i=1,2,3$

with Poisson bracket

$$
\{f, g\}_{S^{2}}=\epsilon_{i j k} \partial^{i} f \partial^{j} g x_{k}
$$

For the coordinates $x^{i}$ it gives the $S O(3)$ algebra

$$
\left\{x_{i}, x_{j}\right\}_{S^{2}}=\epsilon_{i j k} x_{k} \quad i, j, k=1,2,3
$$

The corresponding Hamiltonian flows are, $H \in C^{\infty}\left(S^{2}\right)$,

$$
L_{H}=\epsilon^{i j k} x^{i} \frac{\partial H}{\partial x^{j}} \frac{\partial}{\partial x^{k}} \quad i, j, k=1,2,3
$$

with Lie algebra the area preserving diffeomorphisms of the sphere $\operatorname{SDiff}\left(S^{2}\right)$,

$$
\left[L_{H_{1}}, L_{H_{2}}\right]=L_{\left\{H_{1}, H_{2}\right\}}
$$

With the choice of basis functions for $C^{\infty}\left(S^{2}\right)$, the spherical harmonics $Y_{l m}(\theta, \phi)$, we get the algebra of $\operatorname{SDiff}\left(S^{2}\right)$ Arnold (1978), Hoppe (1982)].

$$
\left\{H_{l m}, H_{l^{\prime} m^{\prime}}\right\}_{S^{2}}=f_{l l^{\prime} l^{\prime \prime}}^{m m^{\prime} m^{\prime \prime}} H_{l^{\prime \prime} m^{\prime \prime}}
$$

Y.Nambu in 1973 Nambu (1973) proposed a generalization of Hamiltonian mechanics for volume preserving flows. The simplest example is for the $M=R^{3}$ case. For a given set $f, g, h \in C^{\infty}\left(R^{3}\right)$ he introduced the 3-bracket

$$
\begin{aligned}
\{f, g, h\} & =\epsilon_{i j K} \partial^{i} f \partial^{j} g \partial^{k} h \\
& =\vec{\nabla} f \cdot(\vec{\nabla} g \times \vec{\nabla} h)
\end{aligned}
$$

which satisfies the integrability equation-Fundamental Identity(FI)

$$
\begin{aligned}
\left\{f_{1}, f_{2},\left\{f_{3}, f_{4}, f_{5}\right\}\right\} & =\left\{\left\{f_{1}, f_{2}, f_{3}\right\}, f_{4}, f_{5}\right\} \\
& +\left\{f_{3},\left\{f_{1}, f_{2}, f_{4}\right\}, f_{5}\right\}+\left\{f_{3}, f_{4},\left\{f_{1}, f_{2}, f_{5}\right\}\right\}
\end{aligned}
$$


L.Takhtajan in [Takhtajan (1994)] showed that the n-bracket defines a hierarchy of $n-1, \cdots, 2$ brackets.

For the $n=3$ for example by fixing the third function $\mathrm{h}$ in (15) we get

$$
\{f, g\}_{h}=\{f, g, h\} \quad \forall f, g \in C^{\infty}\left(R^{3}\right)
$$

which from (16) satisfies the relation

$$
\left\{\left\{f_{1}, f_{2}\right\}_{h}+\left\{\left\{f_{2}, f_{3}\right\}_{h}, f_{1}\right\}_{h}+\left\{\left\{f_{3}, f_{1}\right\}_{h}, f_{2}\right\}_{h}=0\right.
$$

which is the Poisson-Jacobi identity. In this way we may obtain a Poisson bracket for any surface in $R^{3}$, which is defined by the level set function $h\left(x_{1}, x_{2}, x_{3}\right)=$ constant.

If we restrict the variables $x^{1}, x^{2}, x^{3}$ to be on the surface $\mathrm{h}$ then rel. (17) defines on it a non-degenerate Poisson bracket. In effect this surface gets promoted to a phase space with dynamics. As an example we consider the plane $R^{2}$ embedded in $R^{3}$.

1. The Plane $h=\alpha^{i} \dot{x}^{i}, \quad \alpha^{i} \in R$

$$
\left\{x^{i}, x^{j}\right\}_{h}=\epsilon^{i j k} \alpha^{k}
$$

2. The Sphere $x_{1}^{2}+x_{2}^{2}+x_{3}^{2}=1, \quad S^{2} \in R^{3}$

$$
\left\{x^{i}, x^{j}\right\}_{S^{2}}=\epsilon^{i j k} x^{k} \quad S O(3) \text { on } S^{2}
$$

Nambu introduced his mechanics for volume preserving flows in $R^{3}$ by specifying $H_{1}, H_{2} \in C^{\infty}\left(R^{3}\right)$ two Hamiltonians inducing the following eq. of motion

$$
\dot{x}^{i}=\left\{x^{i}, H_{1}, H_{2}\right\}, \quad i=1,2,3
$$

or equivalently

$$
\dot{\vec{x}}=\vec{\nabla} H_{1} \times \vec{\nabla} H_{2}
$$

These equations imply the conservation of the "Hamiltonians"

$$
\begin{aligned}
& \dot{H}_{1}=\dot{\vec{x}} \cdot \vec{\nabla} H_{1}=0 \\
& \dot{H}_{1}=\dot{\vec{x}} \cdot \vec{\nabla} H_{1}=0
\end{aligned}
$$

The ideal example right from Nambu's paper is the Euler's top

$$
\begin{aligned}
& H_{1}=\frac{1}{2}\left(l_{1}^{2}+l_{2}^{2}+l_{3}^{2}\right) \\
& H_{2}=\frac{1}{2}\left(\frac{l_{1}^{2}}{I_{1}}+\frac{l_{1}^{2}}{I_{1}}+\frac{l_{1}^{2}}{I_{1}}\right)
\end{aligned}
$$


with equations of motion

$$
\begin{aligned}
& \dot{l}_{1}=\left(\frac{1}{I_{2}}-\frac{1}{I_{3}}\right) l_{2} l_{3} \\
& \dot{l}_{2}=\left(\frac{1}{I_{3}}-\frac{1}{I_{1}}\right) l_{3} l_{1} \\
& \dot{l}_{3}=\left(\frac{1}{I_{1}}-\frac{1}{I_{2}}\right) l_{1} l_{2}
\end{aligned}
$$

The generalization of the Hamiltonian flow vector fields are the flow vector fields

$$
\begin{aligned}
L_{H_{1}, H_{2}} & =\epsilon^{i j k} \frac{\partial H_{1}}{\partial x_{i}} \frac{\partial H_{2}}{\partial x_{j}} \frac{\partial}{\partial x_{k}} \\
& =\left(\vec{\nabla} H_{1} \times \vec{\nabla} H_{2}\right) \cdot \vec{\nabla}=\vec{v} \cdot \vec{\nabla}
\end{aligned}
$$

where

$$
\dot{\vec{x}}=\vec{v}=\vec{\nabla} H_{1} \times \vec{\nabla} H_{2}
$$

which are volume preserving and therefore describe flows of incompressible fluids. The question, which we pose now, is whether the inverse also holds. Namely if for any flow

$$
\dot{\vec{x}}=\vec{v}(\vec{x})
$$

which is incompressible

$$
\vec{\nabla} \cdot \vec{v}=0
$$

there exist two "Hamiltonians" $H_{1}, H_{2}$ with

$$
v^{i}=\left\{x^{i}, H_{1}, H_{2}\right\}
$$

Since a long time ago Lamb (1932) the answer to this problem has been settled to the affirmative locally in $R^{3}$. Indeed for any incompressible vector flows $\vec{v}$, there is a vector potential $\vec{A}$ such that $\vec{v}=\vec{\nabla} \times \vec{A}$ which, moreover, satisfies the Clebsch-Monge decomposition (CM- stream potentials),

$$
\vec{A}=\vec{\nabla} \alpha+\beta \vec{\nabla} \gamma
$$

from which we can deduce that

$$
\vec{v}=\vec{\nabla} \beta \times \vec{\nabla} \gamma \quad \beta=H_{1}, \gamma=H_{2}
$$

The corresponding infinite dimensional algebra of volume preserving diffeomorphisms in the Clebsch-Monge gauge is Axenides \& Floratos (2007)

$$
\left[L_{H_{1}, H_{2}}, L_{H_{3}, H_{4}}\right]=L_{\left\{H_{1}, H_{2}, H_{3}\right\}, H_{4}}+L_{H_{3},\left\{H_{1}, H_{2}, H_{4}\right\}}
$$


So the full structure of the infinite dimensional group is determined by the 3-bracket algebra of a basis of functions $H_{i} \in C^{\infty}\left(R^{3}\right), \quad i=1,2, \cdots$ Axenides \& Floratos (2009)

$$
\left\{H_{i}, H_{j}, H_{k}\right\}=f_{i j k}^{l} H_{l} \quad i, j, k, l=1,2, \cdots
$$

As an example we present the Nambu 3-algebra on $S^{3}$.

Consider $S^{3}$ isometricallly embedded in $R^{4}$

$$
x_{1}^{2}+x_{2}^{2}+x_{3}^{2}+x_{4}^{2}=1
$$

where $x_{i} \in R, \quad i=1,2,3,4$ The Nambu bracket on $R^{4}$

$$
\left\{x_{i}, x_{j}, x_{k}, x_{l}\right\}_{R^{4}}=\epsilon_{i j k l} \quad i, j, k, l=1,2,3,4
$$

induces a 3-bracket on $S^{3}$. Define : $\forall f_{i} \in C^{\infty}\left(S^{3}\right) \quad i=1,2,3$

$$
\left\{f_{1}, f_{2}, f_{3}\right\}_{S^{3}}=\epsilon^{i j k l} x^{i} \partial^{j} f_{1} \partial^{k} f_{2} \partial^{l} f_{3}
$$

which on the $R^{4}$ coordinates gives

$$
\left\{x_{i}, x_{j}, x_{k}\right\}_{S^{3}}=\epsilon_{i j k l} x_{l} \quad i, j, k, l=1,2,3,4
$$

This is the celebrated Baggert-Lambert algebra for $S O(4)$ Bagger \& Lambert (2007). For any $f, g \in C^{\infty}\left(S^{3}\right)$ we consider the flow vector field

$$
L_{f, g}=\{f, g, \cdot\}_{S^{3}} \quad L_{f, g} h=\{f, g, h\}_{S^{3}}
$$

and thus the Nambu equations on $S^{3}$ are given as :

$$
\dot{x}^{i}=L_{H_{1}, H_{2}} x^{i}=\left\{x^{i}, H_{1}, H_{2}\right\}_{S^{3}} \quad \forall H_{1}, H_{2} \in C^{\infty}\left(S^{3}\right)
$$




\section{The Lorenz Attractor as a Dissipative Nambu Me- chanics}

We are not going to repeat the history of the famous Lorenz-Saltzman system Saltzman(1962), Lorenz(1963)] of nonlinear equations, which is the Galerkin trancation of the Fourier modes of the temperature gradient and stream potential for the Rayleigh-Benard experiment. We will nevertheless present their dynamical system of three variables

$$
\begin{aligned}
\dot{X} & =\sigma(Y-X) \\
\dot{Y} & =X(r-Z)-Y \\
\dot{Z} & =X Y-b Z
\end{aligned}
$$

with $\sigma$ being the Prandl Number, $\mathrm{r}$ the relative Reynolds number and $\mathrm{b}$ is the aspect ratio.

The system has as critical points

1. $X_{1}=Y_{1}=Z_{1}=0$

2. $X_{2}=Y_{2}=\sqrt{b(r-1)}, \quad Z_{2}=r-1$

3. $X_{3}, Y_{3}=-\sqrt{b(r-1)}, \quad Z_{3}=r-1$

Using stability analysis for fixed $\sigma$ and $\mathrm{b}$, their behaviour is controled by the relative Reynolds number $r$. Turbulent behavior starts for values of

$$
r=r_{H}=\sigma \frac{\sigma+b+3}{\sigma-b-1}
$$

with $\left.\sigma=10, \quad b=\frac{8}{3}\left(\rightarrow r_{H}=24.73\right)\right)$. Lorenz's choice is $r=28>r_{H}$ in order to obtain chaotic behaviour [Sparrow (1987)] where Hopf bifurcation appears with $\vec{X}_{1}$ a saddle point with $\vec{X}_{2}$ and $\vec{X}_{3}$ going repelling-unstable.

In our recent work Axenides \& Floratos (2010)] we implemented a decomposition of the Lorenz vector flow into a volume preserving (non-dissipative) and a dissipative components

$$
\vec{v}=\vec{v}_{N D}+\vec{v}_{D}
$$

The non-dissipative component gives a volume preserving flow $\vec{\nabla} \cdot \vec{v}_{N D}=0$

$$
v_{\vec{N} D}=(\sigma Y, X(r-Z), X Y)
$$

with

$$
\begin{aligned}
\dot{X} & =\sigma Y=\left\{X, H_{1}, H_{2}\right\} \\
\dot{Y} & =X(r-Z)=\left\{Y, H_{1}, H_{2}\right\} \\
\dot{Z} & =X Y=\left\{Z, H_{1}, H_{2}\right\}
\end{aligned}
$$


with

$$
\vec{V}_{N D}=\vec{\nabla} H_{1} \times \vec{\nabla} H_{2}
$$

We have identified the conserved Hamiltonians (see also Nevir \& Blender (1994)])

$$
\begin{aligned}
& H_{1}=\frac{1}{2}\left(Y^{2}+(Z-r)^{2}\right) \\
& H_{2}=\sigma Z-\frac{X^{2}}{2}
\end{aligned}
$$

The corresponding Poisson brackets on the surface $\Sigma, H_{2}=$ constant are

$$
\begin{aligned}
\{X, Y\}_{\Sigma} & =\partial_{Z} H_{2}=\sigma \\
\{Y, Z\}_{\Sigma} & =\partial_{X} H_{2}=-X \\
\{Z, X\}_{\Sigma} & =0
\end{aligned}
$$

By eliminating the variables $\mathrm{Y}, \mathrm{Z}$ from eq.(45) we get

$$
\ddot{X}+\left(H_{2}-\sigma r\right) X+\frac{X^{3}}{2}=0
$$

which is just the equation of motion for the one dimensional Anharmonic Oscillator. Depending on the initial conditions we identify its two familiar symmetry phases as:

- $H_{2}-\sigma r \geq 0 \quad$ Single well-Symmetric Phase

- $H_{2}-\sigma r \leq 0 \quad$ Double Well-Broken Phase

By including dissipation we have

$$
\dot{\vec{X}}=\vec{\nabla} H_{1} \times \vec{\nabla} H_{2}-\vec{\nabla} D=\vec{V}_{N D}+\vec{V}_{D}
$$

where the dissipation potential D is given below

$$
\begin{aligned}
H_{1} & =\frac{1}{2}\left((z-r)^{2}+y^{2}\right) \\
H_{2} & =\sigma Z-\frac{X^{2}}{2} \\
D & =\frac{1}{2}\left(\sigma X^{2}+Y^{2}+b Z^{2}\right)
\end{aligned}
$$

Now, of course, $H_{1}, H_{2}$ along with $D$ are not any more conserved. But still $H_{2}$ is a useful quantity since by eliminating $\mathrm{Y}, \mathrm{Z}$ we find the dynamical system for $\mathrm{X}, \mathrm{H}_{2}$

$$
\begin{gathered}
\ddot{X}+(1+\sigma) \dot{X}+X\left(\frac{X^{2}}{2}+H_{2}-\sigma(r-1)\right)=0 \\
\dot{H}_{2}+b H_{2}-\sigma\left(1-\frac{b}{2 \sigma}\right) X^{2}=0
\end{gathered}
$$


The anharmonic oscillator moerover develops a damping term and since $H_{2}$ is not conserved, we obtain a sequence of symmetry breaking and symmetry restoring phases. By solving for $\mathrm{H}_{2}$ we obtain the well known Takeyama memory term in the anharmonic potential Takeyama (1978), Takeyama (1980),

$$
H_{2}(t)=H_{2}(0) e^{-b t}+\sigma\left(1-\frac{b}{2 \sigma}\right) e^{-b t} \int_{0}^{t} d \xi e^{b \xi} X^{2}(\xi)
$$

\section{Matrix Quantization of the Lorenz Attractor}

We will quantize the volume preserving part, in the Nambu form, of the Lorenz attractor system. Firstly we write the Nambu equations,

$$
\begin{aligned}
\dot{X} & =\left\{X, H_{1}\right\}_{H_{2}}=\left\{X, H_{1}, H_{2}\right\} \\
\dot{Y} & =\left\{Y, H_{1}\right\}_{H_{2}}=\left\{Y, H_{1}, H_{2}\right\} \\
\dot{Z} & =\left\{Z, H_{1}\right\}_{H_{2}}=\left\{Z, H_{1}, H_{2}\right\}
\end{aligned}
$$

Using the Poisson algebra of equations (48).

$$
\begin{aligned}
\{X, Y\}_{H_{2}} & =\sigma=\partial_{Z} H_{2} \\
\{Y, Z\}_{H_{2}} & =-X=\partial_{X} H_{2} \\
\{Z, X\}_{H_{2}} & =\partial_{Y} H_{2}=0
\end{aligned}
$$

The quantization of the system is carried through the quantization of the Poisson algebra, i.e. by lifting it to commutators with Weyl-ordered operators

$$
\begin{aligned}
{[\widehat{X}, \widehat{Y}] } & =\imath \hbar \sigma \\
{[\widehat{Y}, \widehat{Z}] } & =-\imath \hbar \widehat{X} \\
{[\widehat{Z}, \widehat{X}] } & =0
\end{aligned}
$$

We must find either infinite dim. matrices or differential operators that satisfy rel. (56) The Quantum Nambu equations for the volume preserving part follow,

$$
\begin{aligned}
\imath \hbar \dot{\hat{X}} & =\left[\widehat{X}, \widehat{H}_{1}\right]_{H_{2}} \\
\imath \hbar \dot{\hat{Y}} & =\left[\widehat{Y}, \widehat{H}_{1}\right]_{H_{2}} \\
\imath \hbar \dot{\widehat{Z}} & =\left[\widehat{Z}, \widehat{H}_{1}\right]_{H_{2}}
\end{aligned}
$$


where the $H_{2}$ index implies that we evaluate the commutators by using the algebra (56) which has as Casimir the second Hamiltonian $\widehat{H}_{2}$ ( parabolic-cylinder phase space)

$$
\widehat{H}_{2}=\frac{\widehat{X}^{2}}{2}-\sigma \widehat{Z}
$$

We obtain

$$
\begin{aligned}
\dot{\hat{X}} & =\sigma \widehat{Y} \\
\dot{\hat{Y}} & =-\frac{1}{2}(\widehat{X} \widehat{Z}+\widehat{Z} \widehat{X})+r \widehat{X} \\
\dot{\widehat{Z}} & =\frac{1}{2}(\widehat{X} \widehat{Y}+\widehat{Y} \widehat{X})
\end{aligned}
$$

from where we get the conservation of $\widehat{H_{1}}, \widehat{H_{2}}$ and a unique time evolution

$$
\begin{aligned}
\widehat{X}(t) & =e^{-\frac{i}{\hbar} t H_{1}} \widehat{X}(0) e^{\frac{i}{\hbar} t H_{1}} \\
\widehat{Y}(t) & =e^{-\frac{i}{\hbar} t H_{1}} \widehat{Y}(0) e^{\frac{i}{\hbar} t H_{1}} \\
\widehat{Z}(t) & =e^{-\frac{i}{\hbar} t H_{1}} \widehat{Z}(0) e^{\frac{i}{\hbar} t H_{1}}
\end{aligned}
$$

By eliminating $\widehat{Y}, \widehat{Z}$ from rels. (59) we get the anharmonic oscillator quantum eqs.

$$
\ddot{\widehat{X}}+\left[\left(\widehat{H}_{2}-\sigma r\right) \widehat{X}+\frac{\widehat{X}^{3}}{2}\right]=0
$$

The $\mathrm{H}_{2}$ Casimir values determine superselection sectors for rel.(61) with $H_{2}<\sigma r$ (Double Well), where quantum tunneling appears. For $H_{2} \geq \sigma r$ (Single Well) anharmonic potential governs quantum dynamics. Both cases possess discrete spectra.

For the non-dissipative part of the quantum Lorenz system, we could also work in the Schrondinger picture for the one dimensional anharmonic potential.

\section{Inclusion of Dissipation; Decoherence of the Quan- tum Lorenz Attractor}

The Quantum Lorenz equations including dissipation are Axenides \& Floratos (2010)

$$
\begin{aligned}
\dot{\hat{X}} & =\sigma(\widehat{Y}-\widehat{X}) \\
\dot{\hat{Y}} & =-\frac{1}{2}(\widehat{X} \widehat{Z}+\widehat{Z} \widehat{X})+r \widehat{X}-\widehat{Y} \\
\dot{\widehat{Z}} & =\frac{1}{2}(\widehat{Y} \widehat{X}+\widehat{X} \widehat{Y})-b \widehat{Z}
\end{aligned}
$$


It is obvious that the quantum algebra(56) does not hold anymore for all times and $\widehat{H}_{1}, \widehat{H}_{2}$ are not conserved. Still we can eliminate $\widehat{Y}, \widehat{Z}$ and obtain a dynamical anharmonic system with quantum Takeyama memory term. The Schrondinger picture is not anymore convenient due to the time nonlocality of the memory term. Qualitatively one expects that weak dissipation will induce a broadening of the discrete energy levels. Moreover for the case of strong dissipation the quantum system is expected to collapse to the classical one.

We can approximate the quantum system through the usage of finite dimensional $N \times$ $N$ Hermitian matrices for the representation of $\widehat{X}, \widehat{Y}, \widehat{Z}$. This is, for example, the case with a spin system with $N=2 s+1$. See, for example, the case in Axenides \& Floratos (2007) of an Euler top interpretation for the Lorenz attractor. Numerical experiments for the non-dissipative systems, show integrable behaviour while for weak dissipation we obtain $\mathrm{N}$ weakly interacting Lorenz attractors. On the other hand for strong dissipation the quantum system quickly undergoes decoherence to a classical system of $\mathrm{N}$ independent Lorenz attractors ( as $\widehat{X}, \widehat{Y}, \widehat{Z}$ become asymptotically mutually commuting).

We evaluated numerically the time evolution of the $\mathrm{N} \times \mathrm{N}$ matrix Lorenz system with the standard parameter values $\left(r=28, \sigma=10, b=\frac{8}{3}\right)$.

We found that the commutators of $\widehat{X}, \widehat{Y}, \widehat{Z}$ undergo rapid decoherence unless dissipation is weak.

$$
\begin{array}{lll}
{[\widehat{X}(t), \widehat{Y}(t)]} & \stackrel{t \rightarrow \infty}{\longrightarrow} e^{-\Gamma_{12} \cdot t}[\widehat{X}(0), \widehat{Y}(0)] \\
{[\widehat{Y}(t), \widehat{Z}(t)]} & \stackrel{t \rightarrow \infty}{\longrightarrow} e^{\Gamma_{23} t}[\widehat{Y}(0), \widehat{Z}(0)] \\
{[\widehat{Z}(t), \widehat{X}(t)]} & \stackrel{t \rightarrow \infty}{\longrightarrow} e^{-\Gamma_{31} t}[\widehat{Z}(0), \widehat{X}(0)]
\end{array}
$$

For completeness we also present the equations for the closed systems of operators $\widehat{H}_{2}, \widehat{X}$

$$
\dot{\hat{H}}_{2}+b \widehat{H}_{2}=\sigma\left(1-\frac{b}{2 \sigma}\right) \widehat{X}^{2}
$$

and

$$
\ddot{\widehat{X}}+(1+\sigma) \dot{\widehat{X}}+\left(\frac{\widehat{X} \widehat{H}_{2}+\widehat{H}_{2} \widehat{X}}{2}-\sigma(r-1) \widehat{X}+\frac{\widehat{X}^{3}}{2}\right)=0
$$

Currently we are in the process of performing numerical calculations for large symmetric or Hermitian marices for the case of weak dissipation where interesting behaviour appears between the quantum and classical overlap regimes. For discussions of the Lorenz attractor quantization in the physical framework of laser instabilities see Elgin \& Sarkar (1984). 


\section{Conclusions-Open Questions Applications}

We presented a framework to quantize non-Hamiltonian dissipative flows in $R^{3}$. We applied the method to the very interesting and rich example of the Lorenz Strange attractor. This we did in order to check the robustness of quantum mechanics against the robustness of strange attractors. The qualitative behavior for a finite matrix approximations of the quantum system, exhibits interesting results for the case of weak dissipation. This is not the case of a typical Lorenz attractor, which exhibits very strong dissipation and undergoes quick decoherence. We believe the study of quantum dynamical systems with soft turbulence Elgin \& Sarkar (1984)] is useful to probe the classical $\leftrightarrow$ quantum regime transition for dissipative dynamical systems.

Applications of quantum turbulence are awaiting for exciting new discoveries and new paradigm shifts in the behaviour of quantum matter and radiation in extreme conditions of high temperatures and densities where the Lagrangian particle concept looses its importance and the Eulerian fluid interpretation takes the lead. In all of these, chaotic attractor mechanisms should prevail. Their quantum mechanical survival will be important to our understanding of quantum turbulence.

In this direction important developments come from recent studies of holographic hydrodynamics in its relation with black hole horizon physics, where the problem of quantization of gravity resurfaces from a new hydrodynamic point of view [Eling et al.(2009)].

\section{References}

[Asakawa et al.(2007)] Asakawa, S. and Bass, A. and Muller, B. [2007] Anomalous transport processes in anisotropically expanding quark-gluon plasmas, Prog. Theor. Phys. 116 p.725 arXiv:hep-ph/0608270.

[ Arnold (1978)] Arnold, V.I. Mathematical Methods of Classical Mechanics [1978] Springer Science.

[ Axenides \& Floratos (2007) ] Axenides, M. and Floratos, E. [2007] Euler top dynamics of Nambu-Goto p-branes JHEP 0703 p. 093, arXiv:hep-th/0608017].

[ Axenides \& Floratos (2009)] Axenides, M and Floratos, E [2009] Nambu-Lie 3-Algebras on Fuzzy 3-Manifolds JHEP 0902 p. 039, arXiv:0809.3493 [hep-th]].

[ Axenides \& Floratos (2010)] Axenides, M. and Floratos, E. [2010] Strange Attractors in Dissipative Nambu Mechanics: Classical and Quantum Aspects, JHEP 1004 p.036, arXiv:0910.3881 [nlin.CD]]. 
[ Axenides \& Floratos (2009)] Axenides, E. and Floratos, E. and Nicolis, S. [2009] Nambu Quantum Mechanics on Discrete 3-Tori J. Phys. A 42 p. 275201, [arXiv:0901.2638 [hep-th]].

[ Axenides (2011) ] Axenides, M.[2011] Non-Hamiltonian Chaos from Nambu Dynamics of Surfaces in Chaos Theory: Modeling, Simulation and Applications, C.H.Skiadas et.al.(Eds), World Scientific Publ.Co., pp 110, arXiv:1109.0470 [nlin.CD]].

[Bagger \& Lambert (2007)] Bagger, J. and Lambert, N. [2007] Gauge Symmetry and Supersymmetry of Multiple M2-Branes Phys. Rev. D 77 p. 065008 , arXiv:0711.0955 [hep-th]].

[ Eckmann \& Ruelle (1985)] Eckmann, J.P. and Ruelle, D. [1985] Ergodic Theory of Chaos and Strange Attractors Review of Modern Physics, vol.57, p. 617.

[ Elgin \& Sarkar (1984) ] Elgin, J.N. and Sarkar, S. [1984] Quantum Fluctuations and the Lorenz Strange Attractor, Phys. Rev. Lett. 52 p. 1215.

[Eling et al.(2009)] Eling,C. and Fouxon,I. and Oz,Y. [2009] The Incompressible Navier-Stokes Equations From Membrane Dynamics Phys. Lett. B 680 p.496, arXiv:0905.3638 [hep-th]].

[Gibson \& Schild (2010)] Gibson, C.H. and Schild, R.H. [2010] Turbulent behavior of protogalaxies at the end of the Plasma Epoch: theory and observation, Jour. of Cosmology 6, p. 1351. arXiv:1004.2016[astro-ph.CO]]

[Hoppe (1982)] Hoppe, J. [1982] Quantum theory of a massless relativistic surface and a two dimensional bound state problem Ph.D. Thesis MIT , Aachen preprint PITHA$86 / 24$.

[Lamb (1932)] Lamb, H. [1932] Hydrodynamics Cambridge U. Press, p.248.

[Landau (1941) ] Landau, L. [1941] J.Phys.5, p.71.

[Landau \& Lifshitz(1987)] Landau, L.D. and Lifshitz, E.M. [1987] Fluid Mechanics, 2nd edit. (Elsevier).

[Lorenz(1963)] Lorenz,E.N. [1963] Deterministic Non-Periodic Flow J.Atm.Sci. 20, p. 130.

[ Nambu (1973)] Nambu, Y. [1973] Generalized Hamiltonian Dynamics Phys. Rev. D 7, p.2405.

[ Nevir \& Blender (1994)] Nevir, P. and Blender, R. [1994] Hamiltonian and Nambu Representation of the Non-Dissipative Lorenz Equation Beitr. Phys. Atmosph. 67, p.133. 
[Saltzman(1962)] Saltzman, B. [1962] Finite Amplitude Free Convection as an Initial Value Problem J. Atm. Sci. 19 p.329.

[Sparrow (1987)] Sparrow, C. [1987] The Lorenz Equation, Bifurcations, Chaos and the Strange Attractors, Springel-Verlag, New York.

[Takhtajan (1994)] Takhtajan, L. [1994] On Foundation Of The Generalized Nambu Mechanics (Second Version) Commun. Math. Phys. 160 , p. 295.

[Takeyama (1978)] Takeyama, K. [1978] Dynamics of the Lorenz Model of Convective Instabilities, Prog.Theor.Phys. 60 p. 613.

[Takeyama (1980)] Takeyama, K. [1980], Prog. Theor. Phys. 63 p.91.

[ Tarasov (2008)] Tarasov, V.E. [2008] Quantum Mechanics of Non-Hamiltonian and Dissipative Systems, Elsevier.

[Van Sciver (2009)] Van Sciver, S.W. and Barenghi, C.F.[2009] Visualization of Quantum Turbulence, Prog.Low Temp.Phys, edt. W.P.Halperin and M.Tsubota .

[ Viana (2000) ] Viana, M. [2000] What's New Lorenz Strange Attractors?, The Mathematical Intelligencer, pp. 6-19.

[Weiss (2008)] Weiss, U. [2008] Quantum Dissipative Systems World Scientific-Series, vol.13. 